\title{
VHDL-AMS based genetic optimization of a fuzzy logic controller for automotive active suspension systems
}

\author{
L. Wang and T. J. Kazmierski \\ School of Electronics and Computer Science \\ University of Southampton, UK \\ $\{\mid w 04 r, t j k\} @ e c s . s o t o n . a c . u k$
}

\begin{abstract}
This paper presents a new type of fuzzy logic controller (FLC) membership functions for automotive active suspension systems. The shapes of the membership functions are irregular and optimized using a genetic algorithm (GA). In this optimization technique, VHDL-AMS is used not only for the modeling and simulation of the fuzzy logic controller and its underlying active suspension system but also for the implementation of a parallel GA. Simulation results show that the proposed FLC has superior performance to that of existing FLCs that use triangular or trapezoidal membership functions.
\end{abstract}

\section{INTRODUCTION}

Automotive suspension systems reduce the vibrations between the sprung and unsprung masses caused by the motion of an automobile so that the vehicle's ride quality could be improved. According to the system's ability to add or extract energy, the suspension systems can be classified as passive, semi-active or active [11]. The passive suspension consists of conventional springs and dampers only. It cannot add energy to the system. The semi-active suspension does not add energy either but changes the damping coefficient of the shock absorbers dynamically to obtain a better suspension quality. The active suspension (Fig.1) contains an actuator, which can generate a force acting on the sprung and unsprung masses, as well as the springs and dampers.

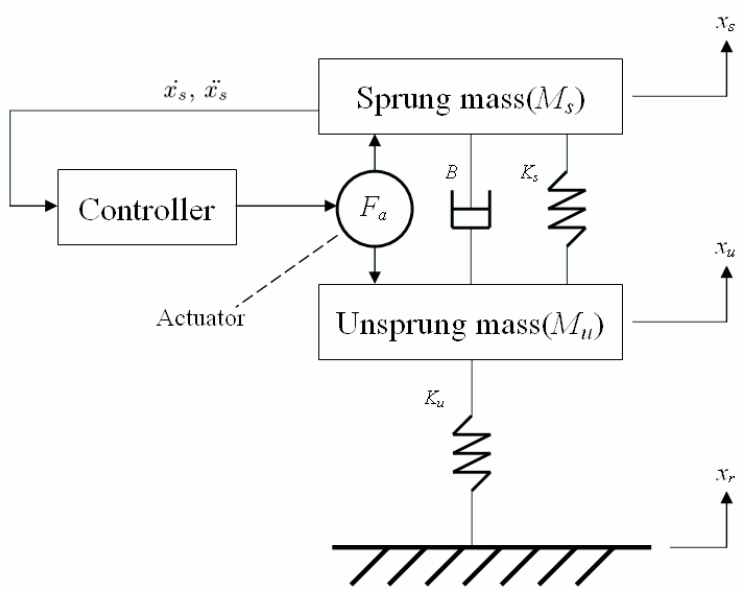

Figure 1: Active suspension system.
The advantages of active suspension systems over passive and semi-active ones have been known for many years [13]. However, the design of a suitable active suspension controller, which determines the value of the actuator force according to the dynamic motions of the sprung and/or unsprung mass, is difficult and still attracts researchers' interest. A number of different control algorithms have been established [15, 11, 2]. Generally speaking, an accurate model of the system is difficult to identify since the input to an active suspension system (i.e. the road displacement) is unpredictable. Fuzzy logic controllers (FLCs), due to their ability of handling uncertain and complex systems, have emerged as a promising technique for high-performance active suspension systems [12, 1]. FLCs are based on the general principles of fuzzy set theory [16] and their input and output variables are similar to a conventional controller. FLC designs reported so far show satisfactory suspension behavior and use fixed-shape, usually triangular or trapezoidal membership functions (Fig.2).

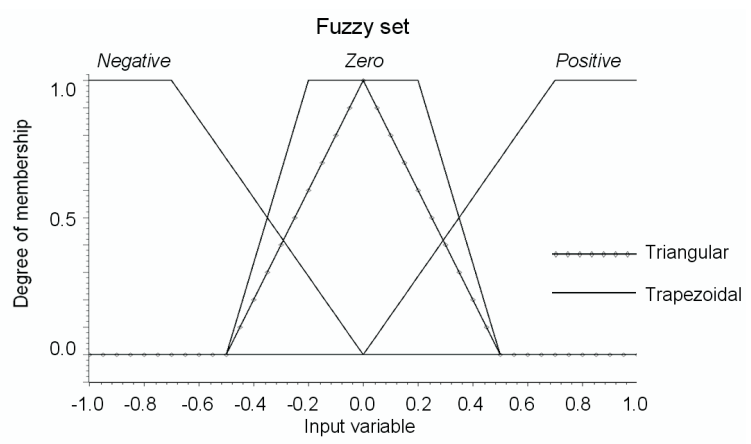

Figure 2: Fuzzy logic triangular and trapezoidal membership functions.

The study presented in this paper attempts to optimize the shape of the membership function to improve the FLC performance. A genetic algorithm, which is an optimization method based on natural selection [4], has been chosen for this purpose. A GA algorithm usually has the following elements: populations of chromosomes, selection according to fitness, crossover to produce new offspring, and random mutation of new offspring [7]. The stochastic nature of GA makes it suitable for fuzzy logic applications.

VHDL-AMS is a hardware description language designed 
to support modeling at various abstraction levels in mixed, electrical and non-electrical physical domains as well as mixed, digital and analogue components [3]. It has been recommended as the unified modeling language for the automotive industry [8]. In this paper we present a model of an entire active suspension system in VHDL-AMS with a parallel GA process implemented in the testbench to optimize the system's FLC. The parallel nature of VHDL-AMS makes the implementation of the GA optimization efficient and straightforward.

\section{ACTIVE SUSPENSION MODEL}

The model shown in Fig.1 is a linear 2-DOF (degree of freedom) quarter-car model. It is simple but contains the basic features of active suspension [14]. The dynamic motions of the sprung and unsprung masses are described by equations (1) and (2) [9] which can be obtained from Newton's second law:

$\ddot{x_{s}} M_{s}=K_{s}\left(x_{u}-x_{s}\right)+B\left(\dot{x_{u}}-\dot{x_{s}}\right)+F_{a}$

$\ddot{x_{u}} M_{u}=-K_{s}\left(x_{u}-x_{s}\right)-B\left(\dot{x_{u}}-\dot{x_{s}}\right)+K_{u}\left(x_{r}-x_{u}\right)-F_{a}$

where $M_{s}$ and $M_{u}$ are vehicle's sprung and unsprung masses, $x_{s}, x_{u}$ and $x_{r}$ are the displacement of sprung mass, unsprung mass and road respectively, $K_{s}$ and $B$ are the coefficients of the passive spring and damper, $K_{u}$ is the tire spring rate and $F_{a}$ is the actuator force. The model acts as the underlying plant of the FLC and is implemented as a VHDL-AMS entity.

\section{SHAPE OPTIMIZATION OF FUZZY LOGIC MEMBERSHIP FUNCTIONS}

In fuzzy logic theory, a membership function is a graphical representation of the input's degree of participation in a fuzzy set. The geometrical shapes of the membership functions used can seriously affect the performance of an FLC. For example, although triangular membership functions are very basic and widely used [12], it was also illustrated that trapezoidal membership functions may generate superior results in certain applications [1] (Fig.2). The optimal shapes of membership functions in an FLC for a specific application may be irregular (see Fig.3) and we show below that they can be calculated by optimization to improve the system's performance. Irregular membership functions are unlikely to lead to more complex hardware implementations given the fact that electronic control units are quite common in today's automobile design.

It has been reported that the optimum choice for active suspension's state variables are the velocity $\dot{x_{s}}$ and acceleration $\ddot{x_{s}}$ of the automobile sprung mass $M_{s}[10]$. So the two variables are chosen as the inputs to the FLC. The output is the actuator force $F_{a}$. The fuzzy sets of the input and output variables are represented by three linguistic variables: positive $(\mathrm{P})$, zero $(\mathrm{Z})$ and negative $(\mathrm{N})$. With these linguistic variables, a set of nine fuzzy rules is developed, as shown in Table 1. These rules were generated by using basic engineering sense. For example, if the velocity is zero and the acceleration is positive then the mass's velocity is going to increase and a negative force should be applied. The fuzzy inference procedure used is the max-product composition and the defuzzification method employed is the center of gravity approach [1].

\begin{tabular}{ccccc}
\multirow{5}{*}{ Velocity } & & \multicolumn{3}{c}{ Acceleration } \\
& & $\mathbf{P}$ & $\mathbf{Z}$ & $\mathbf{N}$ \\
& $\mathbf{Z}$ & $\mathrm{N}$ & $\mathrm{N}$ & $\mathrm{N}$ \\
& $\mathbf{N}$ & $\mathrm{Z}$ & $\mathrm{Z}$ & $\mathrm{P}$ \\
& & & $\mathrm{P}$ & $\mathrm{P}$
\end{tabular}

Table 1: Fuzzy rules base.

\section{GA OPTIMIZATION IN VHDL-AMS TESTBENCH}

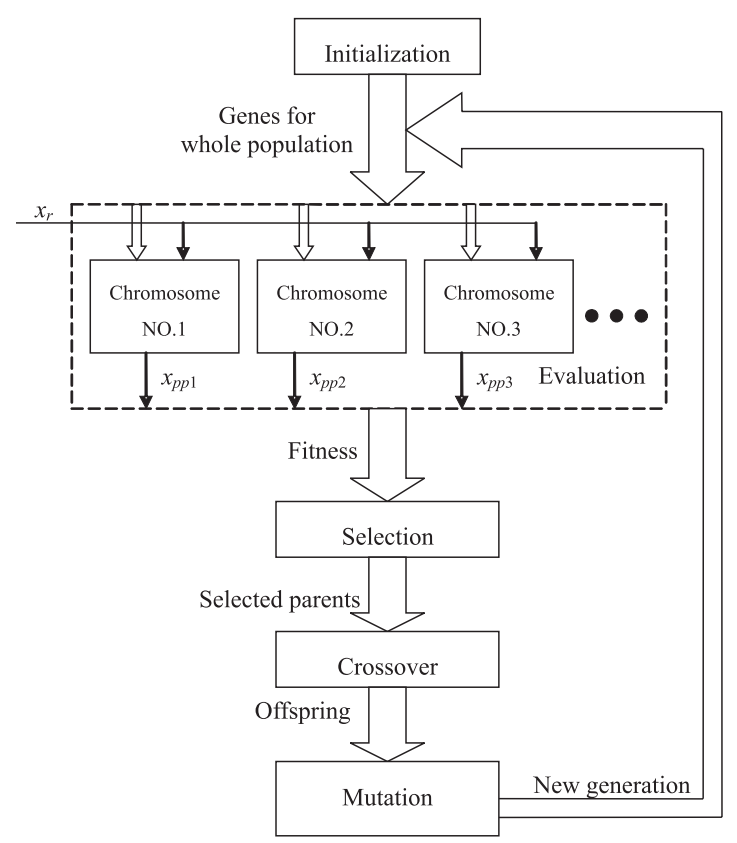

Figure 4: GA optimization in a VHDL-AMS testbench using concurrently running chromosomes.

A flow chart of how the parallel GA is implemented and executed in the VHDL-AMS testbench is shown in Fig.4. In the testbench, instances of the active suspension system, including the FLC, are invoked and each instance represents a chromosome in GA. The shapes of the fuzzy membership functions are the optimization objects. Since the center of gravity method is used for defuzzification, it is only necessary to optimize the membership functions of the input variables. For each of the two input variables, $N$ points from the positive curve and $N$ points from the right half of the zero curve are selected as genes. This is because a membership function is typically symmetrical about the yaxis. These points are equally distributed along the $\mathrm{x}$-axis and their y-values can be adjusted between 0 and 1 . The points are simply connected by straight lines to form piecewise linear membership functions. The optimization goal is to minimize the peak-to-peak value of the sprung mass displacement $x_{s}$ when the system is subject to some kind of stimulus. In the VHDL-AMS implementation, the chromosome is modeled as a component with $4 N$ genes as input parameters, the road displacement $x_{r}$ as the excitation and the peak-to-peak value $x_{p p}$ as the output fitness. Since $x_{p p}$ is a value over a certain time period, a process is needed to track its maximum and minimum value and output the 

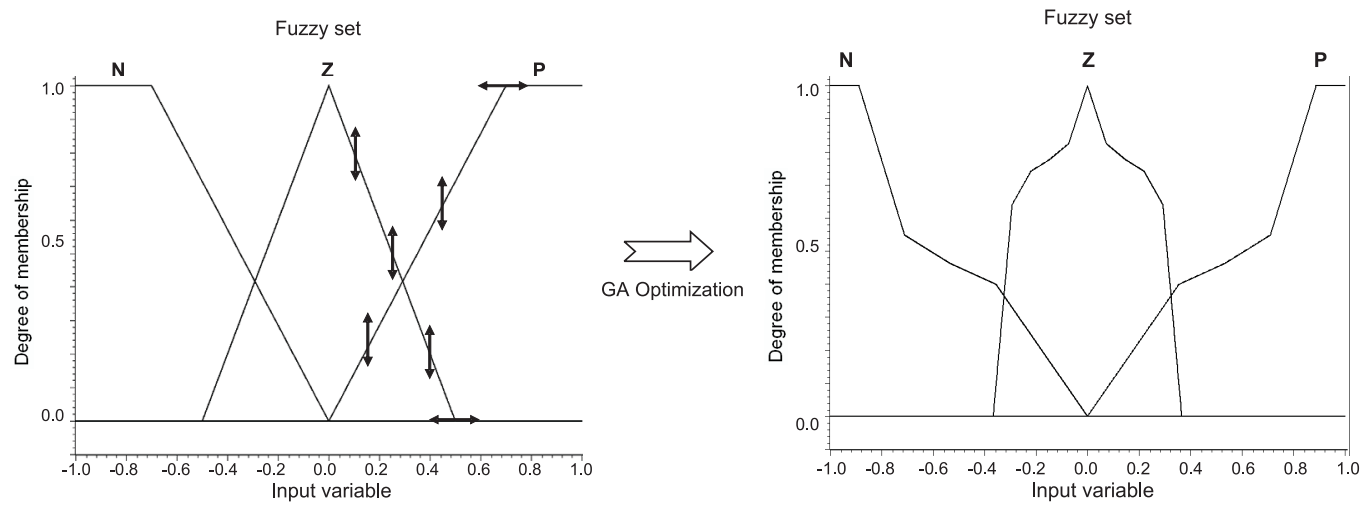

Figure 3: Optimization of the shapes of fuzzy logic membership functions.

peak-to-peak value at the end. Fig.5 is the block diagram of the chromosome. It shows how different components in the entity are connected.

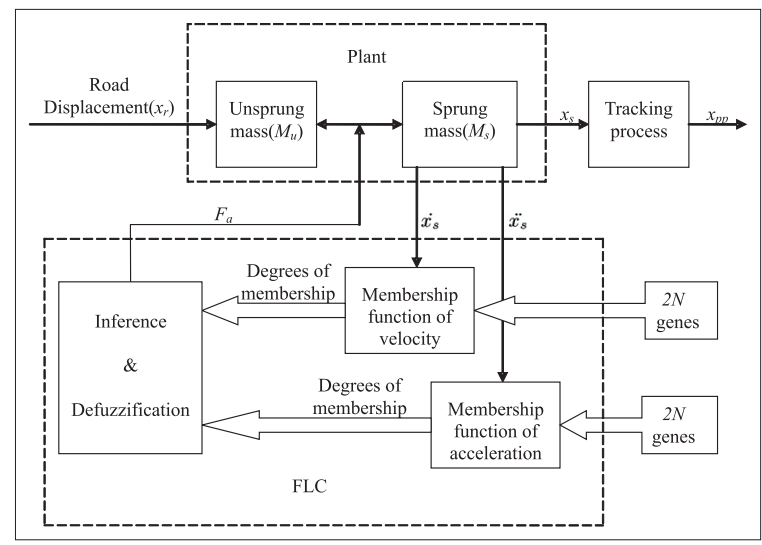

Figure 5: Diagram of one chromosome.

Unlike most existing computer implementations of GA that evaluate one chromosome iteratively to form a population, in the VHDL-AMS based optimization here, the chromosomes of a population are implemented in parallel. The genes are initialized by uniformly distributed random numbers. The same stimulus is applied to the population and all the chromosomes are evaluated simultaneously to get a vector of fitness values. According to the fitness values, the tournament selection is chosen as the parents selection method. Because it prevents premature convergence with efficient computations [7]. The parent with higher fitness (i.e. smaller $x_{p p}$ ) is more likely to be selected to produce offspring. Elitism is also used to improve GA's performance by artificially inserting the best solution into each new generation. Since the genes are real numbers, arithmetic crossover is used to generate the offspring [5]. At last, gene mutation is employed to introduce new solutions into the new population. The evaluation-selection-crossover-mutation process is repeated until all the chromosomes converge to the same fitness. In VHDL-AMS, this loop is controlled by a finite state machine.
Considering the characteristics of the FLC application studied in this paper, a parallel GA can reduce the simulation time thus increase the optimization efficiency. As mentioned above, the fitness of one chromosome can only be obtained through certain time of simulation. If the chromosomes are simulated one by one to evaluate the population, the fitness values need to be maintained before the selection is carried out. Although the previous values do not change, they still use simulator's resources. The parallel evaluation may need less simulation time than a sequential one.

\section{EXPERIMENTAL RESULTS}

In the GA optimization, the number of points on each membership curve $N$ is chosen as 5 . So there are totally 20 genes in one chromosome. The population size is 100 . The crossover and mutation rate are 0.8 and 0.01 respectively. The stimulus is a single sine-wave period jolt of a $10 \mathrm{~cm}$ amplitude and the period of $200 \mathrm{~ms}$. The stimulus is repeated every three seconds, which is the system's settling time. The peak-to-peak value of $x_{s}, x_{p p}$, is also updated every three seconds as the chromosome's fitness. Simulations were carried out using the SystemVision [6] VHDL-AMS simulator from Mentor Graphics. After simulating the testbench for 600 seconds, which corresponds to 200 generations in the GA optimization, the shapes of the membership functions converge (see Fig.6) to an optimum. The simulation CPU time was 16 hours 11 minutes and 44 seconds on a Pentium 4 PC.

The GA-optimized membership functions are implemented in the FLC and simulated. For comparison, the FLCs using triangular and trapezoidal membership functions are also investigated. Simulation results are shown in Fig.7. The peak-to-peak value of road displacement is $20 \mathrm{~cm}$. The conventional FLCs can reduce it to $3.35 \mathrm{~cm}$ (triangular) and $3.55 \mathrm{~cm}$ (trapezoidal). The GA-optimized FLC developed here can further decrease the value to $2.66 \mathrm{~cm}$, which is an improvement of more than $20 \%$.

\section{CONCLUSION}

This paper proposes a VHDL-AMS implementation of parallel GA to optimize the shapes of the fuzzy logic membership functions as a novel way of improving the FLC's performance. A case study of the fuzzy logic controller in an automotive active suspension system has been investigated. 

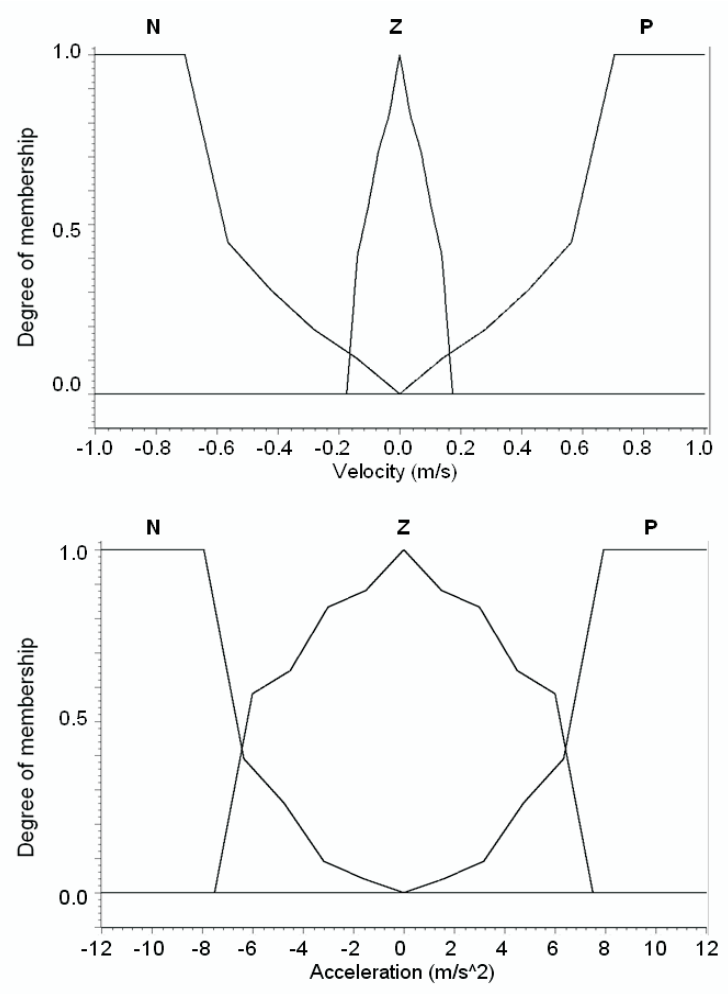

Figure 6: GA optimized membership functions.

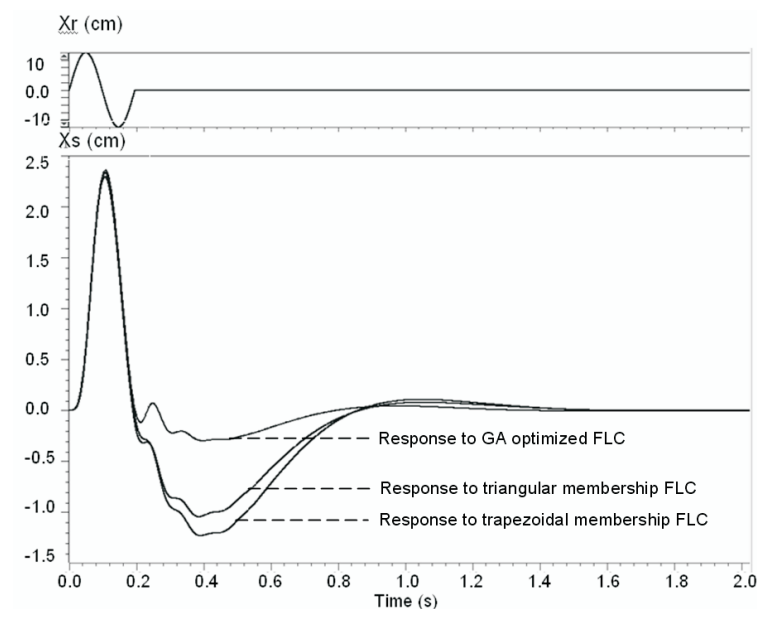

Figure 7: Simulation results.

Simulation results show that the proposed FLC gives superior performance to that of conventional controllers with triangular or trapezoidal membership functions.

\section{REFERENCES}

[1] A. Barr and J. Ray. Control of an active suspension using fuzzy logic. In Fuzzy Systems, 1996. Proceedings of the Fifth IEEE International Conference on, pages 42-48, 8-11 Sept. 1996.

[2] S. Chantranuwathana and H. Peng. Adaptive robust force control for vehicle active suspensions.
International Journal of Adaptive Control and Signal Processing, 18(2):83-102, 2004.

[3] E. Christen and K. Bakalar. VHDL-AMS-a hardware description language for analog and mixed-signal applications. Circuits and Systems II: Analog and Digital Signal Processing, IEEE Transactions on, 46:1263-1272, 1999.

[4] D. Goldberg. Genetic Algorithms in Search, Optimization and Machine Learning. Addison-Wesley Publishing Company, Reading, Massachusetts, 1989.

[5] F. Herrera, M. Lozano, and A. M. Sánchez. A taxonomy for the crossover operator for real-coded genetic algorithms: An experimental study. International Journal of Intelligent Systems, 18:309-338, 2003

[6] Mentor Graphics Corporation. System Vision Users Manual. Version 3.2, Release 2004.3. July 2004.

[7] M. Mitchell. An Introduction to Genetic Algorithms. the MIT Press, Cambridge, Massachusetts, 1996.

[8] E. Moser and N. Mittwollen. VHDL-AMS: the missing link in system design. experiments with unified modelling in automotive engineering. In Design, Automation and Test in Europe, 1998., Proceedings, pages $59-63,1998$.

[9] R. Rajamani and J. Hedrick. Performance of active automotive suspensions with hydraulic actuators: theory and experiment. In American Control Conference, 1994. Proceedings, pages 1214-1218, 29 June-1 July 1994.

[10] P. Ro, C. Kim, and H. Kim. Active suspension using fuzzy logic control. In American Control Conference, 1993. Proceedings, pages 2252-2253, 2-4 June 1993.

[11] Y. Sam, M. Ghani, and N. Ahmad. LQR controller for active car suspension. In TENCON 2000. Proceedings, pages 441-444, 24-27 Sept. 2000.

[12] S.-I. Son and C. Isik. Fuzzy control of an automotive active suspension. In Fuzzy Information Processing Society, 1996. Biennial Conference of the North American, pages 377-381, 19-22 June 1996.

[13] H.-S. Tan and T. Bradshaw. Model identification of an automotive hydraulic active suspension system. In American Control Conference, 1997. Proceedings, pages 2920-2924, 4-6 June 1997.

[14] A. Ulsoy, D. Hrovat, and T. Tseng. Stability robustness of LQ and LQG active suspension. Transactions of ASME, Journal of Dynamic Systems, Measurement, and Control, 116:123-131, 1994.

[15] N. Yagiz, V. Ozbulur, N. Inanc, and A. Derdiyok. Sliding modes control of active suspensions. In Intelligent Control, Proceedings of the 1997 IEEE International Symposium on, pages 349-353, 16-18 July 1997.

[16] L. Zadeh. Fuzzy sets. Information and Control, 8:338-353, 1965. 